\title{
Genetic and immunologic findings in children with recurrent aphthous stomatitis with systemic inflammation
}

\author{
Martina Girardelli ${ }^{1 \dagger}$, Erica Valencic ${ }^{1 \dagger}$, Valentina Moressa ${ }^{1}$, Roberta Margagliotta ${ }^{2}$, Alessandra Tesser ${ }^{1 *}$ (D), \\ Serena Pastore ${ }^{1}$, Ottavia Spadola², Emmanouil Athanasakis' ${ }^{1}$, Giovanni Maria Severini ${ }^{1}$, Andrea Taddio ${ }^{1,2}$ and \\ Alberto Tommasini ${ }^{1,2}$
}

\begin{abstract}
Background: Recurrent aphthous stomatitis with systemic signs of inflammation can be encountered in inflammatory bowel disease, Behçet's disease (BD), Systemic Lupus Erythematosus (SLE). In addition, it has been proposed that cases with very early onset in childhood can be underpinned by rare monogenic defects of immunity, which may require targeted treatments. Thus, subjects with early onset recurrent aphthous stomatitis receiving a clinical diagnosis of BD-like or SLE-like disease may deserve a further diagnostic workout, including immunologic and genetic investigations.
\end{abstract}

Objective: To investigate how an immunologic, genetic and transcriptomics assessment of interferon inflammation may improve diagnosis and care in children with recurrent aphthous stomatitis with systemic inflammation.

Methods: Subjects referred to the pediatric rheumatologist for recurrent aphthous stomatitis associated with signs of systemic inflammation from January 2015 to January 2020 were enrolled in the study and underwent analysis of peripheral lymphocyte subsets, sequencing of a 17-genes panel and measure of interferon score.

Results: We enrolled 15 subjects (12 females, median age at disease onset 4 years). The clinical diagnosis was BD in 8 , incomplete BD in 5, BD/SLE overlap in 1, SLE in 1. Pathogenic genetic variants were detected in 3 patients, respectively 2 STAT1 gain of function variants in two patients classified as BD/SLE overlap and SLE, and 1 TNFAIP3 mutation (A20 haploinsufficiency) in patients with BD. Moreover 2 likely pathogenic variants were identified in DNASE1L3 and PTPN22, both in patients with incomplete BD. Interferon score was high in the two patients with STAT1 GOF mutations, in the patient with TNFAIP3 mutation, and in 3 genetic-negative subjects. In two patients, the treatment was modified based on genetic results.

(Continued on next page)

\footnotetext{
* Correspondence: alessandra.tesser@burlo.trieste.it

${ }^{+}$Martina Girardelli and Erica Valencic contributed equally to this work.

${ }^{1}$ Institute for Maternal and Child Health, IRCCS Burlo Garofolo, Trieste, Italy

Full list of author information is available at the end of the article
}

(c) The Author(s). 2021 Open Access This article is licensed under a Creative Commons Attribution 4.0 International License, which permits use, sharing, adaptation, distribution and reproduction in any medium or format, as long as you give appropriate credit to the original author(s) and the source, provide a link to the Creative Commons licence, and indicate if changes were made. The images or other third party material in this article are included in the article's Creative Commons licence, unless indicated otherwise in a credit line to the material. If material is not included in the article's Creative Commons licence and your intended use is not permitted by statutory regulation or exceeds the permitted use, you will need to obtain permission directly from the copyright holder. To view a copy of this licence, visit http://creativecommons.org/licenses/by/4.0/ The Creative Commons Public Domain Dedication waiver (http://creativecommons.org/publicdomain/zero/1.0/) applies to the data made available in this article, unless otherwise stated in a credit line to the data. 
(Continued from previous page)

Conclusions: Although recurrent aphthous stomatitis associated with systemic inflammation may lead to a clinical diagnosis of BD or SLE, subjects with early disease onset in childhood deserve genetic investigation for rare monogenic disorders. A wider genetic panel may help disclosing the genetic background in the subset of children with increased interferon score, who tested negative in this study.

Keywords: Recurrent aphthous stomatitis, Behçet's disease, Systemic Lupus Erythematosus, Interferon signature, A20 haploinsufficiency, STAT1 mutation

\section{Background}

Recurrent aphthous stomatitis (RAS) is a common and usually isolated and benign complaint in children. However, some cases of RAS present in association with other clinical symptoms or with signs of systemic inflammation, raising a diagnostic challenge to pediatricians. The most common form of RAS with systemic inflammation in pediatrics can be encountered in periodic fever, aphthous stomatitis, pharyngitis, and adenitis syndrome (PFAPA) [1]. The typical periodic recurrence of fever bouts with complete well-being between episodes, and with the absence of significant intestinal or musculoskeletal symptoms, makes PFAPA syndrome an easy diagnosis [2]. Although oral ulcers may present along with PFAPA crises, they are usually smallsized and rapidly self-recovering, never being the patient's major complaint. Aphthous stomatitis accompanying recurrent fevers may also be part of rare syndromes like mevalonate kinase deficiency and Behçet's disease (BD) [3, 4]. In these cases, aphthosis is often severe with multiple painful ulcerations and with slow recovery. RAS associated with raised acute phase reactants can also occur without a clinical story of recurrent fever and in these cases, it should raise the suspicion of inflammatory bowel disease (IBD) [5] or of a rheumatologic condition such as Systemic Lupus Erythematosus (SLE) [6] and BD [4]. However, RAS with systemic inflammation can also be a sign of an immunodeficiency, like HIV infection, neutropenia and monogenic mimics of BD [3, 7-14]. In a recent study, a significant proportion of children with atypical BD was found to have pathogenic mutations in genes associated with monogenic autoinflammatory disorders [15]. Similarly, monogenic disorders can be diagnosed in children who meet the classification criteria for SLE and present recurrent oral ulceration [16-18]. Some of these disorders are associated with dysregulated interferon (IFN)-driven inflammation, which can be revealed by the transcriptomic screening of the IFN signature [19-21]. Of note, an increased IFN signature may be found both in SLE and in BD [22], supporting that rare monogenic conditions with IFN-mediated inflammation may present in an area of clinical and pathological overlap between the two conditions.

The diagnostic challenge is even harder if we consider that some patients with SLE may also meet the classification criteria for pediatric BD [23].
Thus, we decided to investigate if a diagnostic protocol based on immunologic, transcriptomic and next generation sequencing analysis can contribute to improve the classification of subjects with recurrent oral ulcers and systemic inflammation, with possible benefits on the care of the patient in terms of meaningful therapeutic choices and complication surveillance.

\section{Patients and methods Patients}

Subjects consecutively referred to the rheumatology unit of the IRCCS Burlo Garofolo with RAS from January 2015 to January 2020 were enrolled in the study.

As major inclusion criteria were considered the onset before 18 years of age and RAS. Moreover, patients were eligible to be enrolled in the study if they presented at least one of the following signs supportive of systemic inflammation: repeatedly elevated $C$ reactive protein, raised erythrocyte sedimentation rate, genital ulcers, ocular inflammation, arthritis, hepatitis, vasculitis, skin rashes or abscesses. Exclusion criteria were considered: diagnosis of PFAPA, previous diagnosis of a familial periodic fever syndrome, and any refusal to participate in the study by the patient or parents.

For participating in the study, a signed informed consent was obtained from patients' parents/guardians, according to an IRB approved protocol (RC 24/17). All subjects underwent peripheral blood sampling to analyze lymphocyte subsets and to obtain DNA and RNA for genomic and transcriptomic studies. Clinical and laboratory data were recorded in a structured anonymized database.

As regards the initial clinical diagnosis, the case was referred as to $\mathrm{BD}$ when at least three out of six criteria in the pediatric Behçet's Disease (PEDBD) set were met, namely: at least 3 attacks/year of oral aphthosis; genital ulceration typically with scar; skin involvement with folliculitis/acne or erythema nodosum; ocular involvement with uveitis or retinal vasculitis; neurological signs with the exception of isolated headaches; vascular signs as thrombosis or aneurysms [23]. Subjects meeting only 2/ 6 criteria in PEDBD were defined, for the purposes of this study, as possible or incomplete BD (Additional file 2: Table S2). 
Systemic Lupus Erythematosus was classified according to the 1997 revision of ACR criteria [24]. We did not refer to the new EULAR/ACR recommendations for SLE classification [25] since they have not yet been validated in children (Additional file 2: Table S3 and S4).

\section{Flow Cytometry}

\section{Immunophenotypic screening for major immune defects}

Immunophenotype was performed on heparinized peripheral blood samples obtained from each patient by means of flow cytometry. $100 \mu \mathrm{L}$ of blood were stained with two multicolour antibody panels in order to evaluate different lymphocyte subsets, including $B$ cell subpopulations (naïve follicular B cells, marginal zone B cells, switched memory B cells and transitional B cells) and recent thymic emigrants (RTE).

Panel for B cells analysis contained the following antibodies: anti-CD45, anti-CD19, anti-CD38 (Becton Dickinson), anti-CD27, anti-IgM, anti-IgG, anti-CD21, anti-CD10 and anti-IgD (Miltenyi Biotec).

Panel for RTE analysis contained the following antibodies: anti-CD45, anti-CD19, anti-CD4, anti-CD8 (Becton Dickinson), anti-CD3, anti-CD45RA, anti-CD31, anti-CD16 and anti-CD56 (Miltenyi Biotec).

Samples were acquired with MACSQuant Analyzer 10 (Miltenyi Biotec) and analyzed with FlowLogic software (version 7.2.1, Inivai Technologies).

\section{Expression of STAT1 in resting and stimulated peripheral blood cells}

Peripheral blood mononuclear cells (PBMC), obtained from heparinized blood samples by means of density gradient centrifugation, were left unstimulated or were treated with $300 \mathrm{U} / \mathrm{ml}$ of IFN-alpha2a (Miltenyi Biotec). After $24 \mathrm{~h}$ of incubation in $5 \% \mathrm{CO}_{2}$ at $37^{\circ} \mathrm{C}$ cells were recovered, stained with antibodies to cell surface antigens (CD45 (Becton Dickinson), CD3 (Miltenyi Biotec), CD4 (Becton Dickinson)) fixed and permeabilized. PBMC were finally stained with anti-STAT1 antibody (Becton Dickinson) or with an isotype control antibody (Biolegend). Samples were acquired with MACSQuant Analyzer 10 (Miltenyi Biotec) and analyzed with FlowLogic software (version 7.2.1, Inivai Technologies).

\section{IFN signature and IFN score}

Peripheral blood was collected in PAXgene Blood RNA Tubes (PreAnalytiX), extracted with PAXgene Blood RNA Kit (PreAnalytiX) following the manufacturer's instructions, quantified with NanoDrop 2000 Spectrophotometer (Thermo Fisher Scientific) and retro-transcribed using SensiFAST cDNA Synthesis Kit (Bioline).

IFN signature was assessed by calculating the expression of a set of six IFN stimulated genes (IFI27, IFI44L, IFIT1, ISG15, RSAD2 and SIGLEC1), as described elsewhere [26]. Briefly, Real-Time PCR was performed in the AB 7500 Real-Time PCR System (Applied Biosystems), using TaqMan Gene Expression Master Mix (Applied Biosystems) and UPL Probes (Roche). Gene expression was normalized to the amount of reference genes (G6PD and HPRT1) and quantified in respect to a calibrator sample (mix of ten healthy controls) using the $2^{-\Delta \Delta \mathrm{Ct}}$ method. The IFN score (IS) provides the intensity of IFN signature, and it's calculated as the median fold change of the six target genes. IS above 2.466 were considered positive, as previously calculated by Rice et al. to discriminate subjects with IFN-driven monogenic disorders [27].

\section{Genetic analysis}

A custom-made panel for targeted gene panels sequencing (Thermo Fisher Scientific) was designed, including 17 genes listed below: TNFAIP3 (NM_001270508 $\rightarrow$ NP_001257437), STAT1 (NM_007315 $\rightarrow$ NP_009330), DNASE2 (NM $001375 \rightarrow$ NP_001366), DNASE1L3 (NM_004944 $\rightarrow$ NP 004935), TMEM173 (NM_198282 $\rightarrow$ NP_938023), PRKCD $\left(\mathrm{NM} \_006254 \rightarrow \mathrm{NP} \_006245\right)$, TREX1 $\left(\mathrm{NM} \_033629 \rightarrow \mathrm{NP}\right.$ 338599), SAMHD1 (NM_015474 $\rightarrow$ NP_056289), IFIH1 $($ NM_022168 $\rightarrow$ NP_071451), DNASE1 (NM_005223 $\rightarrow$ NP_005214), ISG15 (NM_005101 $\rightarrow$ NP_005092), PTPN22 $\left(\mathrm{NM} \_015967 \rightarrow \mathrm{NP}\right.$ 057051), CTLA4 (NM_005214 $\rightarrow$ NP 005205), STAT4 (NM_003151 $\rightarrow$ NP_003142), TLR4 $\left(\mathrm{NM}_{-}\right.$ $138554 \rightarrow$ NP_612564), $\quad$ RORC $\quad($ NM_005060 $\rightarrow$ NP $005051), R C 3 H 1$ (NM_001300850 $\rightarrow$ NP_001287779).

Gene selection was based on lists of genes known to be associated with SLE and/or type I monogenic interferonopathies, BD or BD like disease (OMIM, Orphanet andPubMed databases).

All exons and flanking introns are covered 100\%, the panel size is $62.58 \mathrm{~kb}$ and 2 pools of primers overlay the entire coding region referred to by the Human hg38 genome. DNA libraries were generated using Ion Ampliseq Library kit 2.0 (Thermo Fisher Scientific) according to manufacturer's protocol, purified with magnetic bead technology Agencourt AMPure XP (Beckman Coulter) and quantified with KAPA Library Quantification Kits (Roche). The sequencing step was performed on the Ion TorrentTM PGM platform (Thermo Fisher Scientific). The signal processing was analyzed by the Torrent SuiteTM software v5.12 and the output file was further annotated using wANNOVAR free software (http:// wannovar.wglab.org/).

All the obtained variants were filtered according the following criteria: minor allele frequency (MAF) $(<0.02$ if recessive inheritance model or $<0.001$ if dominant inheritance model), type of mutation (non-synonymous, nonsense, frameshift, splicing about 10 nucleotides from the splice site), damaging prediction and phenotype correlation. 
Database used for MAF prediction was gnomAD browser (https://gnomad.broadinstitute.org/). In case of missense mutations, we considered those presenting a pathogenic prediction in at least two of the four in silico prediction tools Polyphen-2, SIFT, LRT and Mutation Taster, in addition to a high CADD score $(>15)$ and GERP score (Genomic Evolutionary Rate Profiling) as a measure of the conservation of the genomic position [28-33]. Human Gene Mutation Database professional (HGMD) was used to define association with mutation and specific phenotype.

Variants considered to be causative were validated by Sanger Sequencing both in proband and parents, when available.

\section{Results}

\section{Patients' characteristics}

We enrolled 15 subjects (12 females, median age at disease onset 4 years, range $0.3-15$ years). Recurrent aphthous stomatitis was a presenting symptom in all patients. The initial diagnosis was $\mathrm{BD}$ in 8 , incomplete Behçet Disease (BD-i) in 5, BD/SLE overlap in 1, SLE in 1. Clinical characteristics are shown in Table 1, together with the results of laboratory investigations.

\section{Immunophenotypic analysis}

Immunophenotypic analysis showed only mild alterations of uncertain significance in subject $\# 5$, who showed an increased percentage of NK cells (23.3\%) and low B cells (5.0\%). No significant alteration was found in the other patients as concerns the main lymphocyte subset, including B cell subpopulations and recent thymic emigrants (RTE) (Additional file 1: Table S1).

\section{Genetic analysis}

Pathogenic variants were detected in 3 patients, respectively 2 gain of function (GOF) mutation in STAT1 (\#1 with BD/SLE overlap, \#10 with SLE) and 1 TNFAIP3 mutation (A20 haploinsufficiency, \#15 with BD). Additionally, 2 likely pathogenic variants were identified respectively in DNASE1L3 (\#5, BD-i) and PTPN22 (\#2 with BD-i) genes (Table 2).

The pathogenic role of variants was assessed by online bioinformatic tools and, in the case of STAT1 GOF variants was also studied by the analysis of expression of the protein in peripheral blood cells. As shown in Fig. 1, patient \#1 did not display a clear increase in STAT1 expression compared with the control. However, he carried the p.N574T variant, which lies in the same amino acid position previously described in association to a gain of function mutation of the protein and is similarly predicted to have a pathogenic effect [37]. Patient \#10, who had the previously reported mutation p.T288A [36], showed a constitutive hyperexpression of STAT1 that was only slightly increased by IFN-alpha stimulation.

\section{IFN score}

IFN score was above the positivity cut-off in 6 subjects. Results are compared with measures in 20 subjects with stomatitis associated with non-monogenic conditions (10 patients with PFAPA and 10 with IBD), which tested negative. Two of them were classified as SLE (\#10) or BD/SLE overlap (\#1), 4 as BD (\#9, \#15) or incomplete BD (\#8, \#12) (Table 2). Three patients among those with high IS had positive genetic results $\# 1$ and \#10 with STAT1 GOF mutations, \#15 with TNFAIP3 mutation, Fig. 2).

\section{Clinical follow-up in subjects diagnosed with a monogenic disorder}

The results from this study affected the follow up of patients for various aspects. In two patients, previously classified as affected with SLE or BD/SLE overlap (\#1 and \#10), the diagnosis was changed in STAT1 GOF disease and in one patient (\#15), previously diagnosed with BD, the diagnosis was changed in A20 haploinsufficiency.

Based on the genetic results and on the presence of a high IS in these three patients, a treatment with JAK inhibitor could be reasonable, and indeed patient \#10 started low dose baricitinib, in addition to mofetil mycophenolate and colchicine. After 1 month of treatment, mycophenolate was discontinued and in the following 2 months she didn't experience recurrence of infections of aphthous stomatitis.

In the two patients with STAT1 GOF, at follow-up more attention was put to the prevention and early treatment of infections, considering that they can present a severe course in this syndrome.

In patient \#15, with A20 haploinsufficiency, a treatment with adalimumab was started, considering the high risks of disease progression without treatment and the colchicine intolerance. At the last follow-up, after 6 months of treatment, the patient didn't refer further recurrence of aphthae, abscesses and fever crisis, even if laboratory investigations still revealed an increased IS. Of note, the patient \#15 mother, also carrying the A20 mutation, was also affected with BD not requiring specific medications, whilst a treatment with adalimumab is being considered for the grandmother, who presents complaints similar to the nephew.

The follow-up care of patients carrying mutation in DNASE1L3 and PTPN22 so far was not affected by the genetic diagnosis.

Similarly, the finding of increased IS in other three patients without genetic mutations had so far, no obvious consequence on their clinical care. 
Girardelli et al. Pediatric Rheumatology $\quad$ (2021) 19:70

Page 5 of 10

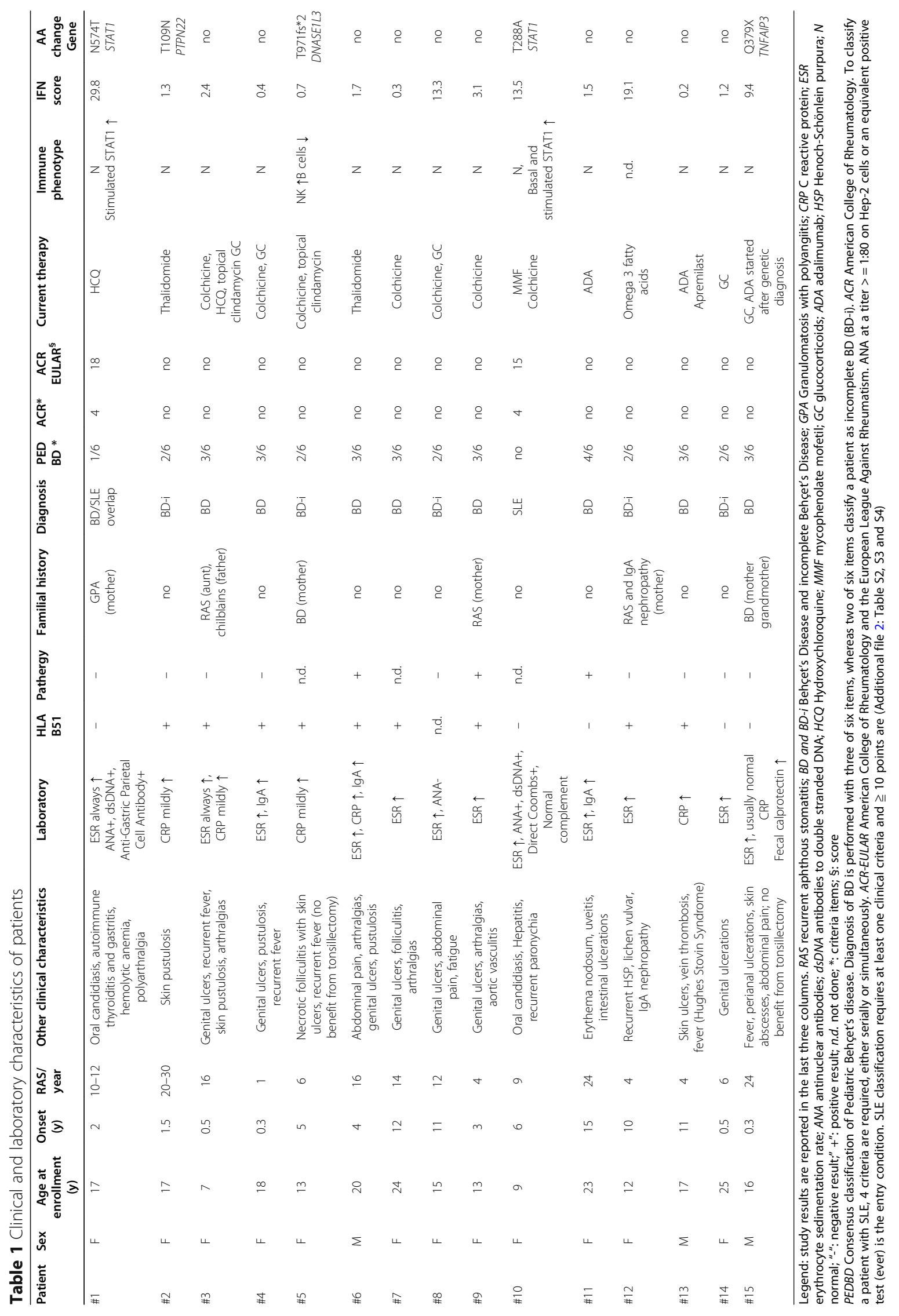


Table 2 Genetic characteristics of identified mutations

\begin{tabular}{|c|c|c|c|c|c|c|c|c|c|c|c|c|c|}
\hline $\mathrm{Pt}$ & Gene & Inheritance & Chr & Variant & $\begin{array}{c}\text { dbSNP } \\
\text { (Reference) }\end{array}$ & gnomAD & GERP & CADD & SIFT & PP2 & LRT & MT & $\begin{array}{l}\text { STATUS } \\
\text { (carrier) }\end{array}$ \\
\hline$\# 1$ & STAT1 & $A D$ & $2 q 32.2$ & c.1721A > C p.N574T & na & na & 5.73 & 22.7 & $\mathrm{~T}$ & $\mathrm{D}$ & $\mathrm{D}$ & $\mathrm{D}$ & $\begin{array}{c}\mathrm{HZ} \\
\text { (De novo) }\end{array}$ \\
\hline \#2 & PTPN22 & $\mathrm{AD} / \mathrm{mt}$ & $1 \mathrm{p} 13.2$ & c.326C > A p.T109N & rs771337900 & 0.00001 & 5.41 & 27.2 & $\mathrm{D}$ & $\mathrm{D}$ & $\mathrm{D}$ & $\mathrm{D}$ & $\begin{array}{c}\mathrm{HZ} \\
\text { (mother) }\end{array}$ \\
\hline \#5 & DNASETL3 & $A R$ & $3 p 14.3$ & c.288_289delCA p.T97lfs*2 & $\begin{array}{c}\text { rs } 751206379 \\
{[34,35]}\end{array}$ & 0.00008 & na & na & na & na & na & na & $\begin{array}{l}\mathrm{HZ} \\
\text { (na) }\end{array}$ \\
\hline \#10 & STAT1 & $A D$ & $2 q 32.2$ & c. $862 A>$ G p.T288A & $\begin{array}{c}\text { rs387906765 } \\
{[36]}\end{array}$ & na & 4.5 & 23.1 & $\mathrm{D}$ & $P$ & $\mathrm{~N}$ & $\mathrm{D}$ & $\begin{array}{c}\mathrm{HZ} \\
\text { (De novo) }\end{array}$ \\
\hline \#15 & TNFAIP3 & $A D$ & $6 \mathrm{q} 23.3$ & c.C1135T p.Q379X & na & na & 5.63 & 39 & na & na & $\mathrm{D}$ & $\mathrm{D}$ & $\begin{array}{c}\mathrm{HZ} \\
\text { (mother) }\end{array}$ \\
\hline
\end{tabular}

Legend. gnomAD refers to total allele frequency (exomes and genomes); $A D$ autosomal dominant; $A R$ autosomal recessive; $m t$ multifactorial disease; GERP Genomic Evolutionary Rate Profiling; CADD Combined Annotation-Dependent Depletion; SIFT Sorting Intolerant From Tolerant; PP2 Polyphen-2; LRT Likelihood Ratio Test; $M T$ Mutation Taster; $T$ tolerated; $D$ damaging/deleterious; $P$ possibly damaging; $N$ neutral; $H Z$ indicate heterozygous condition; $n a$ not available The "variant" column shows cDNA sequence and Protein (amino acid) change referring to the coordinates of the gene transcript reported in the materials and methods

\section{Discussion}

Recurrent aphthous stomatitis (RAS) in children is usually a benign idiopathic disorder requiring only supportive care. However, in some cases RAS may be a presenting sign of a systemic disorder.

Patients may seek the attention of a rheumatologist because of early onset of complaints in infancy, high frequency of episodes, severity of oral ulcers, or due to the presence of a systemic inflammatory involvement, as revealed by laboratory examinations of by inflammatory symptoms involving other organs (such as genital ulcers, vasculitis, hepatitis, skin rashes and abscesses). As discussed further, these patients should undergo a clinical whole exome sequencing targeted to immune dysregulation genes.

The most common diagnosis in children with RAS and systemic inflammation is probably PFAPA syndrome [38]. However, RAS is rarely a serious issue in patients with PFAPA, where mouth ulcers are small, shallow, scattered or solitary, seemingly not painful; and frequently only are noted in episodes subsequent to the query; in contrast with other autoinflammatory syndrome where RAS is usually symptomatic and associated with systemic symptoms. In PFAPA genital ulcers are exceptionally reported, and the prognosis is usually good with prompt response to on demand glucocorticoids and complete healing with growth or after tonsillectomy [39]. Relapsing after tonsillectomy is a rare event, which may raise the suspicion of distinct disorders, such as Hereditary Periodic Fever syndromes [40, 41], or which may forecast a progression toward $\mathrm{BD}$, as suggested by retrospective interviews performed in adulthood [42]. For this reason, we excluded subjects with PFAPA from our study, but included patients with a PFAPA-like onset whose disease progressed after tonsillectomy. Apart from PFAPA, the most common cause of RAS and inflammation is BD [43]. In these cases, oral and genital ulcerations are often a serious complaint. The positive pathergy test can help in the BD diagnosis, but it is not considered a criterion because in some populations, as
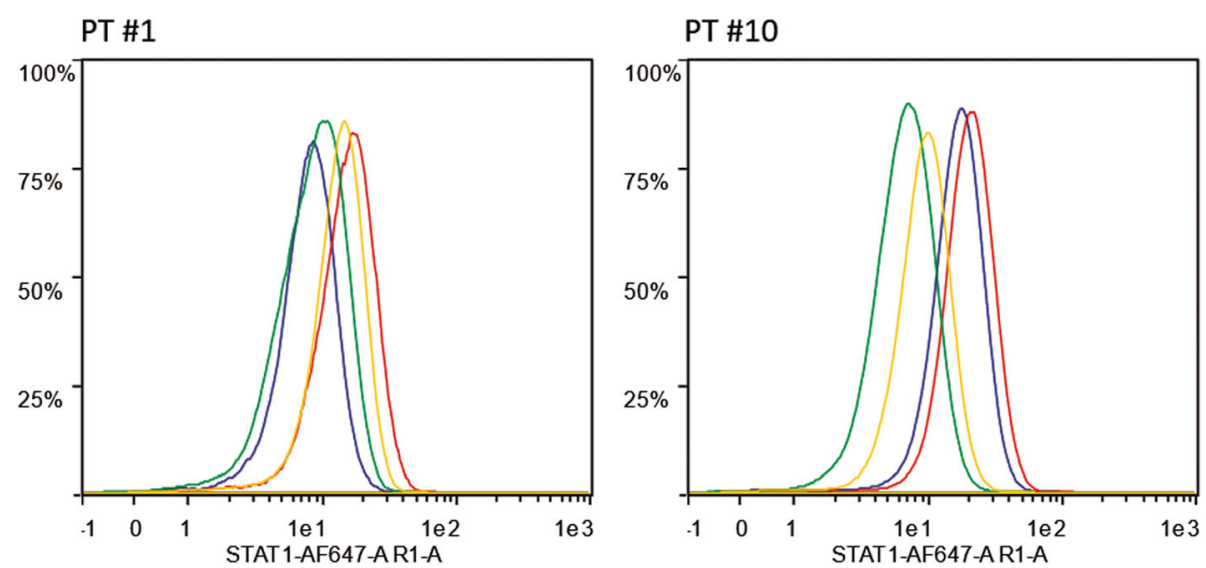

Fig. 1 STAT1 expression. STAT1 expression in CD4 T cells from patient \#1 and \#10. Blue and Red: resting and IFN-alpha stimulated cells from patients; Green and Yellow: resting and IFN-alpha stimulated cells from healthy controls 


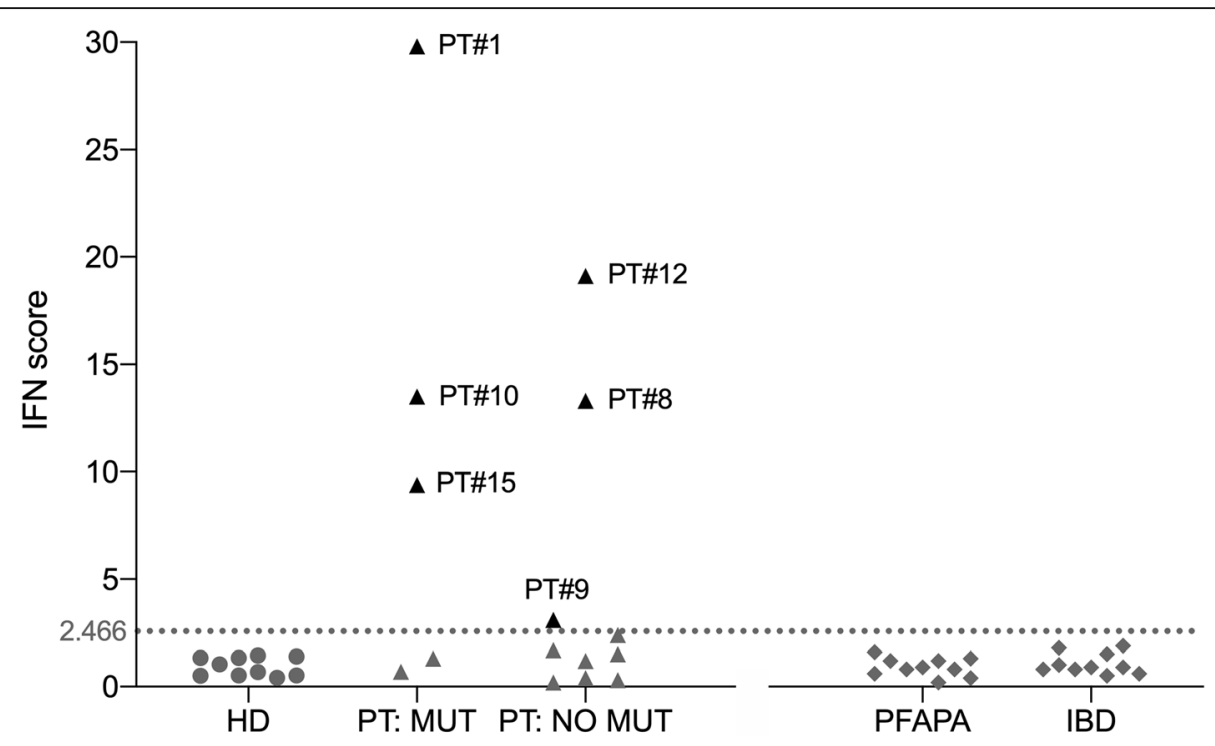

Fig. 2 IFN score. IFN score (IS) in healthy controls (HC) and patients (PT), compared with subjects with PFAPA and IBD. The graphical display of IS is grouped in $\mathrm{HC}$ (n. 10, grey circle), patients (positive IS: black triangle; negative IS: grey triangle), with ("PT: MUT", n. 5) and without genetic mutations ("PT: NO MUT", n. 10), and subjects with PFAPA (n. 10, grey rhombus) and IBD (n. 10, grey rhombus). The dashed light-grey line represents the cut-off value (2.466) determined by Rice et al. [27]

in our series, can be infrequent. However, BD is quite rare in pediatrics and the onset of the disease in younger children should raise the suspicion of an underlying genetic disorder [4]. In agreement, a recent study in a pediatric series of BD showed that a high proportion of patients carried pathogenic mutations in genes associated with monogenic autoinflammatory disorders [15]. In our series, all cases carrying a pathogenic mutation in the analyzed genes had a disease onset before the age of 6 years, with an average age at onset of 3 years, which is very young and below the mean age of onset for BD (7 years) or SLE (11 years). Indeed, early onset may identify a subset of disease with severe phenotype which is likely to be enriched for genetic variants.

RAS with systemic inflammation may also be found in children with SLE. In these cases, too, a stronger genetic component may be found compared with adult-onset disease. For example, a recent Israeli study based on whole exome sequencing in childhood-onset SLE, showed that a high proportion of subjects carried pathogenic mutations associated with monogenic SLE [18]. Of note, there is a considerable overlap between some cases of pediatric SLE and BD and accordingly some of the causative genes may underlie both conditions, as in the case of STAT1 gain of function mutations (GOF) and A20 haploinsufficiency [44].

Interestingly, oral ulceration associated with SLE is usually painless, multiple, nonspecific, and most common sites are tongue and labial mucosa.

In our series we detected two cases as well with STAT1 GOF mutation. As expected, both patients had complained of mucosal candidiasis, which should have raised the suspicion of a primary immunodeficiency like STAT1 GOF. However, in both cases, candidiasis was initially just considered as a complication of mucositis. Indeed, candida infections have been widely reported in association with SLE mucositis, even if we cannot know if any of the cases described in the literature were actually due to STAT1 GOF $[45-49,50,51]$. Interestingly, one of our patients with STAT1 GOF had received a diagnosis of SLE, meeting the ACR classification criteria, whilst the other one had a diagnosis of $\mathrm{BD} / \mathrm{SLE}$ overlap disorder. The patient with A20 haploinsufficiency had a diagnosis of $\mathrm{BD}$ based on PEDDB criteria, but he also had some features more typical of SLE, such as increased IFN signature.

In typical cases, diagnosis of BD or SLE is a process driven by the clinical rheumatologist experience taking into account clinical and laboratory items. In atypical cases, classification criteria can be used to reinforce the diagnosis. However, there is general agreement that both diseases are heterogeneous entities encompassing distinct disorders characterized by systemic autoimmunity. It is still not clear whether the detection of monogenic forms accounting for particular phenotypes will reflect on better therapeutic choices and improved follow-up care in these cases.

In our series, the two patients with STAT1 GOF had a partial control of the disease on treatment with mycophenolate mofetil and colchicine in the first case and with hydroxychloroquine in the second one. However, after the genetic diagnosis, low dose baricitinib was 
proposed to both patients to improve the control of symptoms. Moreover, given the high risk of severe infections in STAT1 GOF disease, more attention was paid on the need for treating any bacterial or fungal infection with systemic antifungal or antibiotic therapy.

In the patient with A20 haploinsufficiency, who had a recurrent-relapsing disorder since his first years of life, a treatment with adalimumab was started with the aim of stopping inflammatory recurrence, as treatment with colchicine was refused because of intolerance. Of note, according to the literature, A20 haploinsufficiency may have distinctive clinical features compared with $\mathrm{BD}$, such as early-onset, familial occurrence, recurrent fever attacks, gastrointestinal involvement, and infrequent ocular involvement, which can influence follow up and therapeutic choices [52].

It is noteworthy that the patient with A20 haploinsufficiency, similarly to other three subjects with BD or incomplete BD, had a high IS. Indeed, A20 haploinsufficiency may present some clinical overlap with SLE, which is more typically associated with increased IS [53]. For example, antinuclear and anti-DNA antibodies can be measured in almost half patients with A20 haploinsufficiency [54]. Moreover, a recent study showed that among subjects with A20 haploinsufficiency, those with a high IS may have a greater benefit from treatment with JAK inhibitors [20]. On the contrary, the role of IFNs in sporadic $\mathrm{BD}$ is less defined. Even if increased IFN production can be observed in a proportion of monocytes from patients with BD [55], IFN-mediated inflammation is not actually a common finding in BD. This concept is even reinforced by the notion that patients with $\mathrm{BD}$ uveitis may be treated with the administration of IFN-alpha. Thus, whether a high IS may identify a particular disease subset in BD, is an interesting issue to be addressed in future studies, with a wider set of genes to be investigated.

Clinical pictures overlapping distinct rheumatologic conditions are common in adults but are much rarer in pediatrics. Our experience supports the new paradigm that every child with an overlapping rheumatological phenotype should be studied for a rare monogenic condition.

In addition to STAT1 and TNFAIP3, we found also likely pathogenic variants in PTPN22 and DNASE1L3 in two patients with incomplete BD. Although PTPN22 polymorphisms have been considered as susceptibility factors for BD or SLE, data are controversial and may be affected by distinct population settings [56, 57]. Biallelic DNASE1L3 deficiency is associated with early-onset familial SLE $[58,59]$ and hypocomplementemic urticarial vasculitis syndrome [34, 60]. Heterozygous hypomorphic mutations in DNASE1L3 have been described in subjects with SLE anti-DNA antibodies sensitive to DNASE1L3 digestion [35]. However, no sign of SLE was present in our patient and the association with a BD-like phenotype in our case is of unclear significance.

The high percentage of monogenic cases in our cohort, is probably due to a bias in patient selection. Our Institute is considered one of the national research centers of rare diseases that attracts complex cases not solved in other institutes.

Is also important underline that we did not identify any patient with RAS and inflammatory bowel disease; this is probably a referral bias, notably three of patients reported were previously referred to our Gastroenterology unit.

\section{Conclusions}

RAS with systemic inflammation may lead to a clinical diagnosis of BD or SLE, moreover patients with early disease onset deserve genetic investigation for rare monogenic disorders. Pediatricians should be aware that RAS can drive the suspicion of a monogenic disorder diagnosis if onset is very early and there are signs of organ involvement that cannot be attributed certainty to a specific polygenic disease.

The IS can be elevated in a subset of patients with BD who sometimes also present clinical elements of overlap with SLE. However, a wider genes panel should be investigated in the subset of children with increased IS but negative for genetic analyses. Integration of laboratory data can lead to a correct disease classification and significant improvements in therapy and follow-up.

\section{Abbreviations}

BD: Behçet's disease; BD-i: Incomplete Behçet's disease; SLE: Systemic Lupus Erythematosus; RAS: Recurrent aphthous stomatitis; PFAPA: Periodic fever, aphthous stomatitis, pharyngitis and adenitis syndrome; IBD: Inflammatory bowel disease; GOF: Gain of function

\section{Supplementary Information}

The online version contains supplementary material available at https://doi. org/10.1186/s12969-021-00552-y.

\section{Additional file 1.}

Additional file 2.

\section{Acknowledgements}

Not Applicable.

\section{Authors' contributions}

MG, EV, ATesser designed the experiments. MG, EV, RM, ATesser carried out the laboratory analysis. VM, SP, OS, ATaddio, ATommasini contributed to evaluate the clinical features. EA, GMS critically analyzed the obtained results. ATommasini wrote the first draft of the paper. All authors contributed substantially to article editing and approved the final manuscript as submitted and take full responsibility for it.

\section{Funding}

The study was funded by the Institute for Maternal and Child Health IRCCS Burlo Garofolo, grant \#24/17 and the Italian Ministry of Health, grant \#RF2016-02362384. 


\section{Availability of data and materials}

The datasets used and/or analysed during the current study are available from the corresponding author on reasonable request.

\section{Declarations}

\section{Ethics approval and consent to participate}

A signed informed consent was obtained from patients' parents/guardians, according to an IRB approved protocol (RC 24/17).

\section{Consent for publication}

An institutional consent form is available.

\section{Competing interests}

None of the Authors has any conflict of interests regarding the matters treated in the manuscript.

\section{Author details}

'Institute for Maternal and Child Health, IRCCS Burlo Garofolo, Trieste, Italy. ${ }^{2}$ University of Trieste, Trieste, Italy.

\section{Received: 27 October 2020 Accepted: 14 April 2021 Published online: 10 May 2021}

\section{References}

1. Thomas KT, Feder HM Jr, Lawton AR, Edwards KM. Periodic fever syndrome in children. J Pediatr. 1999;135:15-21.

2. Gattorno M, Caorsi R, Meini A, Cattalini M, Federici S, Zulian F, et al. Differentiating pfapa syndrome from monogenic periodic fevers. Pediatrics. 2009;124:e721-8.

3. Ter Haar NM, Jeyaratnam J, Lachmann HJ, Simon A, Brogan PA, Doglio M, et al. The phenotype and genotype of mevalonate kinase deficiency: a series of 114 cases from the eurofever registry. Arthritis Rheumatol. 2016;68: 2795-805

4. Kone-Paut I. Behcet's disease in children, an overview. Pediatr Rheumatol Online J. 2016;14:10.

5. Lankarani KB, Sivandzadeh GR, Hassanpour S. Oral manifestation in inflammatory bowel disease: a review. World J Gastroenterol. 2013;19:8571-9.

6. Chiewchengchol D, Murphy R, Edwards SW, Beresford MW. Mucocutaneous manifestations in juvenile-onset systemic lupus erythematosus: a review of literature. Pediatr Rheumatol Online J. 2015;13:1.

7. Wolach B, Scharf Y, Gavrieli R, de Boer M, Roos D. Unusual late presentation of $\mathrm{X}$-linked chronic granulomatous disease in an adult female with a somatic mosaic for a novel mutation in cybb. Blood. 2005:105:61-6.

8. Erdos M, Jakobicz E, Soltesz B, Toth B, Bata-Csorgo Z, Marodi L. Recurrent, severe aphthous stomatitis and mucosal ulcers as primary manifestations of a novel stat1 gain-of-function mutation. Front Immunol. 2020;11:967.

9. Pfajfer L, Mair NK, Jimenez-Heredia R, Genel F, Gulez N, Ardeniz O, et al. Mutations affecting the actin regulator wd repeat-containing protein 1 lead to aberrant lymphoid immunity. J Allergy Clin Immunol. 2018;142:1589-604 e11.

10. Feske S, Gwack Y, Prakriya M, Srikanth S, Puppel SH, Tanasa B, et al. A mutation in orai1 causes immune deficiency by abrogating crac channel function. Nature. 2006;441:179-85.

11. Huck K, Feyen O, Niehues T, Ruschendorf F, Hubner N, Laws HJ, et al. Girls homozygous for an il-2-inducible t cell kinase mutation that leads to protein deficiency develop fatal ebv-associated lymphoproliferation. J Clin Invest. 2009;119:1350-8.

12. Sorte HS, Osnes LT, Fevang B, Aukrust P, Erichsen HC, Backe PH, et al. A potential founder variant in carmil2/rltpr in three norwegian families with warts, molluscum contagiosum, and t-cell dysfunction. Mol Genet Genomic Med. 2016:4:604-16.

13. Lalaoui N, Boyden SE, Oda H, Wood GM, Stone DL, Chau D, et al. Mutations that prevent caspase cleavage of ripk1 cause autoinflammatory disease. Nature. 2020;577:103-8.

14. Zhou Q, Wang H, Schwartz DM, Stoffels M, Park YH, Zhang Y, et al. Loss-offunction mutations in tnfaip3 leading to a20 haploinsufficiency cause an early-onset autoinflammatory disease. Nat Genet. 2016;48:67-73.

15. Papadopoulou C, Omoyinmi E, Standing A, Pain CE, Booth C, D'Arco F, et al. Monogenic mimics of behcet's disease in the young. Rheumatology (Oxford). 2019;58:1227-38.
16. Demirkaya E, Sahin S, Romano M, Zhou Q, Aksentijevich I. New horizons in the genetic etiology of systemic lupus erythematosus and lupus-like disease: monogenic lupus and beyond. J Clin Med. 2020;9.

17. Rice GI, Rodero MP, Crow YJ. Human disease phenotypes associated with mutations in trex1. J Clin Immunol. 2015;35:235-43.

18. Tirosh I, Spielman S, Barel O, Ram R, Stauber T, Paret G, et al. Whole exome sequencing in childhood-onset lupus frequently detects single gene etiologies. Pediatr Rheumatol Online J. 2019;17:52.

19. Kaleviste E, Saare M, Leahy TR, Bondet V, Duffy D, Mogensen TH, et al. Interferon signature in patients with stat1 gain-of-function mutation is epigenetically determined. Eur J Immunol. 2019;49:790-800.

20. Schwartz DM, Blackstone SA, Sampaio-Moura N, Rosenzweig S, Burma AM, Stone D, et al. Type i interferon signature predicts response to jak inhibition in haploinsufficiency of a20. Ann Rheum Dis. 2020;79:429-31.

21. Zimmermann N, Wolf C, Schwenke R, Luth A, Schmidt F, Engel K, et al. Assessment of clinical response to janus kinase inhibition in patients with familial chilblain lupus and trex1 mutation. JAMA Dermatol. 2019:155:342-6.

22. Puccetti A, Fiore PF, Pelosi A, Tinazzi E, Patuzzo G, Argentino G, et al. Gene expression profiling in behcet's disease indicates an autoimmune component in the pathogenesis of the disease and opens new avenues for targeted therapy. J Immunol Res. 2018;2018:4246965.

23. Kone-Paut I, Shahram F, Darce-Bello M, Cantarini L, Cimaz R, Gattorno M, et al. Consensus classification criteria for paediatric behcet's disease from a prospective observational cohort: Pedbd. Ann Rheum Dis. 2016;75:958-64.

24. Hochberg MC. Updating the american college of rheumatology revised criteria for the classification of systemic lupus erythematosus. Arthritis Rheum. 1997:40:1725.

25. Aringer M, Costenbader K, Daikh D, Brinks R, Mosca M, Ramsey-Goldman R, et al. 2019 European league against rheumatism/American College of Rheumatology classification criteria for systemic lupus erythematosus. Ann Rheum Dis. 2019;78:1151-9.

26. Pin A, Monasta L, Taddio A, Piscianz E, Tommasini A, Tesser A. An easy and reliable strategy for making type i interferon signature analysis comparable among research centers. Diagnostics (Basel). 2019;9.

27. Rice Gl, Forte GM, Szynkiewicz M, Chase DS, Aeby A, Abdel-Hamid MS, et al. Assessment of interferon-related biomarkers in aicardi-goutieres syndrome associated with mutations in trex1, rnaseh2a, rnaseh2b, rnaseh2c, samhd1, and adar: a case-control study. Lancet Neurol. 2013;12:1159-69.

28. Adzhubei IA, Schmidt S, Peshkin L, Ramensky VE, Gerasimova A, Bork P, et al. A method and server for predicting damaging missense mutations. Nat Methods. 2010;7:248-9.

29. Kumar P, Henikoff S, Ng PC. Predicting the effects of coding non-synonymous variants on protein function using the SIFT algorithm. Nat Protoc. 2009;4:1073-81.

30. Chun S, Fay JC. Identification of deleterious mutations within three human gen- omes. Genome Res. 2009;19:1553-61.

31. Schwarz JM, Rödelsperger C, Schuelke M, Seelow D. MutationTaster evaluates disease-causing potential of sequence alterations. Nat Methods. 2010;7:575-6.

32. Kircher M, Witten DM, Jain P, O'Roak BJ, Cooper GM, Shendure J. A general framework for estimating the relative pathogenicity of human genetic variants. Nat Genet. 2014:46:310-5.

33. Cooper GM, Goode DL, Ng SB, Sidow A, Bamshad MJ, Shendure J, et al. Single-nucleotide evolutionary constraint scores highlight disease-causing mutations. Nat Methods. 2010;7:250-1.

34. Carbonella A, Mancano G, Gremese E, Alkuraya FS, Patel N, Gurrieri F, et al. An autosomal recessive dnase1/3-related autoimmune disease with unusual clinical presentation mimicking systemic lupus erythematosus. Lupus. 2017; 26:768-72.

35. Johannes Hartl, Robert M. Clancy, Peter M. Izmirly2, H. Michael Belmont1, Nicole Kaiden3, Nicole Bornkamp, et al. Dysfunction of the DNAS E1L3 Pathway and Antigen Accumulation in Lupus Nephritis [abstract]. 2018 ACR/ARHP Annual Meeting.

36. Liu L, Okada S, Kong XF, Kreins AY, Cypowyj S, Abhyankar A, et al. Gain-offunction human stat1 mutations impair il-17 immunity and underlie chronicmucocutaneous candidiasis. J Exp Med. 2011;208:1635-48.

37. Giovannozzi S, Lemmens V, Hendrix J, Gijsbers R, Schrijvers R. Live cell imaging demonstrates multiple routes toward a stat1 gain-of-function phenotype. Front Immunol. 2020;11:1114

38. Hofer M, Pillet $\mathrm{P}$, Cochard MM, Berg S, Krol P, Kone-Paut I, et al. International periodic fever, aphthous stomatitis, pharyngitis, cervical adenitis syndrome cohort: Description of distinct phenotypes in 301 patients. Rheumatology (Oxford). 2014:53:1125-9. 
39. Batu ED. Periodic fever, aphthous stomatitis, pharyngitis, and cervical adenitis (pfapa) syndrome: Main features and an algorithm for clinical practice. Rheumatol Int. 2019;39:957-70.

40. De Pieri C, Taddio A, Insalaco A, Barbi E, Lepore L, Ventura A, et al. Different presentations of mevalonate kinase deficiency: a case series. Clin Exp Rheumatol. 2015;33:437-42.

41. Pehlivan E, Adrovic A, Sahin S, Barut K, Kul Cinar O, Kasapcopur O. Pfapa syndrome in a population with endemic familial mediterranean fever. J Pediatr. 2018;192:253-5.

42. Cantarini L, Vitale A, Bersani G, Nieves LM, Cattalini M, Lopalco G, et al. Pfapa syndrome and behcet's disease: a comparison of two medical entities based on the clinical interviews performed by three different specialists. Clin Rheumatol. 2016:35:501-5.

43. Gurkan A, Ozlu SG, Altiaylik-Ozer P, Kurtul BE, Karacan CD, Senel S. Recurrent aphthous stomatitis in childhood and adolescence: a single-center experience. Pediatr Dermatol. 2015;32:476-80.

44. Li G, Li Y, Liu H, Shi Y, Guan W, Zhang T, et al. Genetic heterogeneity of pediatric systemic lupus erythematosus with lymphoproliferation. Medicine (Baltimore). 2020;99:e20232.

45. Sieving RR, Kauffman CA, Watanakunakorn C. Deep fungal infection in systemic lupus erythematosus - three cases reported, literature reviewed. J Rheumatol. 1975:2:61-72.

46. Wasserman AM, Sarantopoulos GP, Khanna D. Fungal leukocytoclastic vasculitis as a presentation of systemic vasculitis in a patient with systemic lupus erythematosus. J Clin Rheumatol. 2009;15:383-6.

47. Chen $G L$, Chen Y, Zhu CQ, Yang CD, Ye S. Invasive fungal infection in Chinese patients with systemic lupus erythematosus. Clin Rheumatol. 2012;31:1087-91.

48. Fangtham M, Magder LS, Petri MA. Oral candidiasis in systemic lupus erythematosus. Lupus. 2014;23:684-90.

49. Silva MF, Ferriani MP, Terreri MT, Pereira RM, Magalhães CS, Bonfá E, et al. A multicenter study of invasive fungal infections in patients with childhoodonset systemic lupus Erythematosus. J Rheumatol. 2015;42:2296-303.

50. Chowdhry IA, Tan IJ, Mian N, Mackay M, Keiser H, Davidson A. Systemic lupus erythematosus presenting with features suggestive of human immunodeficiency virus infection. J Rheumatol. 2005;32:1365-8.

51. Febronio MV, Pereira RMR, Bonfa E, Takiuti AD, Pereyra EAG, Silva CCA. Inflammatory cervicovaginal cytology is associated with disease activity in juvenile systemic lupus erythematosus. Lupus.2007;16:430-5.

52. Tsuchida N, Kirino Y, Soejima Y, Onodera M, Arai K, Tamura E, et al. Haploinsufficiency of a20 caused by a novel nonsense variant or entire deletion oftnfaip3 is clinically distinct from behcet's disease. Arthritis Res Ther. 2019;21:137.

53. Baechler EC, Batliwalla FM, Karypis G, Gaffney PM, Ortmann WA, Espe KJ, et al. Interferon-inducible gene expression signature in peripheral blood cells ofpatients with severe lupus. Proc Natl Acad Sci U S A. 2003;100:2610-5.

54. Aeschlimann FA, Batu ED, Canna SW, Go E, Gul A, Hoffmann P, et al. A20 haploinsufficiency (ha20): clinical phenotypes and disease course of patients with anewly recognised nf-kb-mediated autoinflammatory disease. Ann Rheum Dis. 2018;77:728-35.

55. Flint SM, Jovanovic V, Teo BW, Mak A, Thumboo J, McKinney EF, et al. Leucocyte subset-specific type 1 interferon signatures in sle and other immunemediated diseases. RMD Open. 2016;2:e000183.

56. Bahrami T, Valilou SF, Sadr M, Soltani S, Salmaninejad A, Soltaninejad E, et al. Ptpn22 gene polymorphisms in pediatric systemic lupus erythematosus. Fetal Pediatr Pathol. 2020;39:13-20.

57. Tizaoui K, Kim SH, Jeong GH, Kronbichler A, Lee KS, Lee KH, et al. Association of ptpn22 1858c/t polymorphism with autoimmune diseases: a systematic review and bayesian approach. J Clin Med. 2019;8.

58. Batu ED, Kosukcu C, Taskiran E, Sahin S, Akman S, Sozeri B, et al. Whole exome sequencing in early-onset systemic lupus erythematosus. J Rheumatol. 2018:45:1671-9.

59. Al-Mayouf SM, Sunker A, Abdwani R, Abrawi SA, Almurshedi F, Alhashmi N, et al. Loss-of-function variant in dnase1/3 causes a familial form of systemic lupus erythematosus. Nat Genet. 2011;43:1186-8.

60. Ozcakar ZB, Foster J 2nd, Diaz-Horta O, Kasapcopur O, Fan YS, Yalcinkaya F, et al. Dnase1/3 mutations in hypocomplementemic urticarial vasculitis syndrome. Arthritis Rheum. 2013;65:2183-9.

\section{Publisher's Note}

Springer Nature remains neutral with regard to jurisdictional claims in published maps and institutional affiliations.

Ready to submit your research? Choose BMC and benefit from:

- fast, convenient online submission

- thorough peer review by experienced researchers in your field

- rapid publication on acceptance

- support for research data, including large and complex data types

- gold Open Access which fosters wider collaboration and increased citations

- maximum visibility for your research: over $100 \mathrm{M}$ website views per year

At BMC, research is always in progress.

Learn more biomedcentral.com/submissions 